\section{Christian Chabannon Patrice Mannoni}

\title{
Les cellules souches hématopoiétiques du sang périphérique chez l'homme
}

Les cellules souches du sang périphérique (CSSP) sont des produits de cytaphérèse, obtenus chez des malades ayant reçu une chimiothérapie aplasiante ou un facteur de croissance hématopoïétique recombinant, tels le GM-CSF ou le G-CSF. La proportion de véritables cellules souches hématopoiétiques dans ces populations cellulaires est beaucoup plus faible que celle de progéniteurs plus mûrs ou de cellules différenciées. Elles procurent cependant une reconstitution hématopoiétique souvent plus rapide que celle obtenue après transplantation de moelle osseuse et permettent une réduction de la morbidité associée à la chimiothérapie à très forte dose appliquée aux malades atteints de cancers de mauvais pronostic. Les nouveaux procédés de sélection des progéniteurs, les cultures liquides avec des cytokines ("expansion in vitro"), vont contribué à élargir les applications de la transplantation de cellules souches hématopoiétiques. De plus, ces cellules sont une cible intéressante pour les tentatives de thérapie génique.

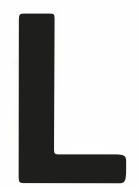

es populations de cellules différenciées et fonctionnelles identifiables dans les organes et tissus hématopoiétiques et le sang périphérique dérivent d'une population de cellules souches qui ne représentent qu'une infime fraction du tissu hématopoïétique. Le passage de la cellule souche hématopoiétique aux cellules mûres se fait par étapes successives où interviennent les phénomènes de survie et de mort cellulaire, de prolifération et de différenciation. Ces phénomènes sont sous la dépendance de signaux régulateurs complexes, incluant de nombreuses inter- actions cellulaires et des molécules solubles, ou cytokines, dont un nombre croissant a été identifié au cours des dernières années.

Durant l'ontogenèse, les premières cellules hématopoïétiques identifiables apparaissent au seizième jour de gestation au niveau du tissu extraembryonnaire. Les premières cellules souches hématopoïétiques peuvent être mises en évidence dans le foie entre la quatrième et la cinquième semaine de gestation et pourraient dériver des cellules extra-embryonnaires après migration à travers la circulation ombilicale. A partir de la dixième semaine de gestation, les cel- 


\section{RÉFÉRENCES}

1. Faucher C, Le Corroller AG, Blaise D, et al. Comparison of G-CSF primed peripheral blood progenitor cells (PBPC) and bone marrow auto-transplantation : clinical assessment and cost-effectiveness. Bone Marrow Transplant 1995 (sous presse).

2. Jordan CT, Lemischka IR. Clonal and systemic analysis of long-term hematopoiesis in the mouse. Genes Dev $1990 ; 4: 220-32$.

3. Brenner MK, Hill DR, Holladay MS, et al. Gene marking to determine whether autologous marrow infusion restores long-term haemopoiesis in cancer patients. Lancet $1993 ; 342: 11347$.

4. O'Shaughnessy JA, Cowan KH, Wilson W, et al. Pilot study of ICE (ifosfamide, carboplatin, etoposide) chemotherapy and autologous bone marrow transplant (ABMT) with neoR-transduced bone marrow and peripheral blood stem cells in patients with metastatic breast cancer. Hum Gene Ther $1993 ; 4: 331-54$.

5. Blanchet JP, Mouchiroud G. Les cellules souches hématopoiétiques : une population plus hétérogène qu'il n'y paraît. médecine/ sciences 1993; 9 ; 959-63.

6. Hatzfeld J, Panterne B, Sansilvestri P, et al. La cellule souche hématopoiétique humaine: du mythe à la réalité. médecine/ sciences $1993 ; 9$ : 1110-2.

7. Vaziri P, Dragowska W, Allsopp RC, Thomas TE, Harley CB, Lansdorp PM. Evidence for a mitotic clock in human hematopoietic stem cells : loss of telomeric DNA with age. Proc Natl Acad Sci USA 1994; 91 : 9857-60.

8. McNiece IK, Stewart FM, Deacon DM, et al. Detection of a human CFC with a high proliferative potential. Blood 1989; 74:609pro.

9. Leary AG, Ogawa M. Blast cell colony assay for umbilical cord blood and adult bone marrow progenitors. Blood 1987; 69 : 953-6.

10. Udomsakdi C, Lansdorp PM, Hogge DE, Reid DS, Eaves AC, Eaves CJ. Characterization of primitive hematopoietic cells in normal human peripheral blood. Blood 1992 ; lules hématopoïétiques colonisent le tissu osseux qui restera le site exclusif de l'hématopoïèse après la naissance. Il existe cependant, chez l'enfant et chez l'adulte, un compartiment de cellules souches circulantes dont la signification physiologique n'est pas définitivement établie.

Les cellules souches hématopoïétiques (CSH) les mieux étudiées en termes de propriétés fonctionnelles et de phénotype sont celles de la moelle osseuse. L'attention des scientifiques et des médecins s'est récemment attachée à la présence de cellules souches hématopoïétiques dans d'autres tissus - sang périphérique, sang du cordon ombilical, tissus fœtaux - et aux applications thérapeutiques possibles. La présence de progéniteurs hématopoïétiques dans le sang périphérique est connue depuis plusieurs décennies grâce aux expériences de circulation croisée chez l'animal, et à la mise en évidence de cellules clonogéniques et de cellules reconstituant l'hématopoïèse chez l'animal et chez l'homme. Cependant, la fréquence de ces cellules à l'état d'homéostasie est très faible, plus encore que dans la moelle osseuse. La découverte que cette fréquence pouvait augmenter chez des patients sortant de la phase d'aplasie succédant à une chimiothérapie, ou après administration d'un facteur de croissance de l'hématopoïèse, a stimulé les applications cliniques et l'étude de la biologie des cellules souches du sang périphérique (CSSP). Rapidement, avant même que l'ensemble des questions scientifiques ait été résolu, les CSSP ont été utilisées de façon croissante et tendent actuellement à supplanter les greffons médullaires. Produit "transfusionnel" tout autant que "transplant", elles ont modifié certains aspects de la pratique médicale dont les conséquences scientifiques, thérapeutiques, éthiques et économiques méritent une évaluation [1]. Parce que leur recueil est aisé, elles sont au coeur de débats parfois contradictoires où elles apparaissent tantôt comme un "médicament " aux multiples possibilités, tantôt comme une population cellulaire à l'avenir thérapeutique limité. Quoi qu'il en soit, elles sont aujourd'hui un composant important des premières tentatives de manipulation cellulaire ex vivo.
L'identification des cellules souches du sang périphérique se heurte aux mêmes difficultés que l'identification des cellules souches de la moelle osseuse ou d'autres tissus hématopoïétiques

La conception hiérarchique de l'hématopoièse prévoit deux propriétés cardinales pour les cellules souches hématopoïétiques : la capacité de se différencier et celle de s'autorenouveler. I a propriété de différenciation vers les lignées myéloïdes et lymphoïdes est utilisée pour identifier une population contenant des cellules souches hématopoïétiques chez l'animal : c'est la capacité de repeupler la moelle (marrow repopulating ability, MRA) après un traitement myéloablatif. En situation allogénique ou congénique, il est possible d'établir que les cellules lymphoïdes $\mathrm{B}$ et $\mathrm{T}$ dérivent des cellules souches hématopoïétiques présentes dans le greffon tout comme les cellules myéloïdes. L'utilisation du polymorphisme d'intégration d'un marqueur rétroviral permet de démontrer que la reconstitution hématopoḯtique au long terme chez des souris ayant reçu un traitement myélo-ablatif est oligoclonale [2] ; ces résultats sont confirmés par le modèle allophénique. Chez l'homme, les données sur la reconstitution hématopoïétique in vivo ont été acquises en situation thérapeutique. Si la contribution d'un greffon médullaire à la reconstitution myéloïde et lymphoïde a été bien établie lors de transplantations allogéniques, et plus récemment après marquage des cellules d'un greffon autologue par intégration du gène de résistance à la néomycine [3], il n'en est pas de même pour les CSSP. En effet, l'expérience clinique accumulée au cours des dernières années l'a été exclusivement en situation autologue; des programmes de transplantation allogénique avec des CSSP, et des protocoles de marquage de CSSP autologues sont actuellement mis en place [4] et devraient permettre de connaître la contribution des CSSP à la reconstitution myéloïde et lymphoïde, à court et à long terme.

L'autorenouvellement des cellules souches hématopoḯtiques est une hypothèse qui repose sur les capacités du système hématopoïétique à 
produire quotidiennement plusieurs milliards de cellules fonctionnelles, pendant toute la vie d'un individu et sans épuisement malgré les agressions que peuvent représenter un accident hémorragique ou infectieux sévère, la récupération hématologique après une chimiothérapie aplasiante, ou le prélèvement, éventuellement répété, de moelle osseuse dans le cadre d'une transplantation médullaire. Dans ce modèle, une au moins des deux cellules filles issues de la division d'une cellule souche hématopoïétique conserve toutes les propriétés fonctionnelles de la première. En réalité, la définition précise de la cellule souche hématopoiétique humaine n'étant pas achevée $[5,6]$, il est difficile d'affirmer que le produit d'une division est strictement identique à la cellule mère. En l'absence de test expérimental pour autorenouvellement, il est difficile de démontrer que cette hypothèse est correcte et de l'utiliser pour mettre en évidence des cellules souches hématopoïétiques au sein d'une population cellulaire. Les délétions des oligonucléotides répétitifs des extrémités télomériques des chromosomes, mises en évidence par le groupe de Peter Lansdorp, suggèrent au contraire que les populations des progéniteurs hématopoïétiques les plus immatures subissent aussi un processus de sénescence [7] ; ces observations confortent l'hypothèse alternative selon laquelle la population des cellules souches hématopoïétiques totipotentes n'est pas inépuisable, au moins chez l'animal ou chez l'homme adulte, ainsi que le suggéraient déjà les expériences de transplantations successives chez la souris.

Les progéniteurs hématopoiétiques déjà engagés dans le processus de différenciation et qui constituent la descendance des cellules souches hématopoïétiques peuvent être mis en évidence grâce à plusieurs tests fonctionnels

La mise en évidence de ces progéniteurs repose sur l'utilisation de tests fonctionnels qui ont été élaborés en travaillant essentiellement avec des cellules médullaires. Ces techniques ont été appliquées à l'étude du sang périphérique. C'est le cas, par $\mathrm{m} / \mathrm{s} n^{\circ} \mathrm{l}$, vol. 11 , janvier 95 exemple, des tests clonogéniques: décrits au cours des années 1960, d'abord chez la souris puis, rapidement, chez l'homme, ces tests mettent en évidence des cellules dont la propriété est de donner naissance à des colonies en milieu semi-solide (agar ou méthyl cellulose) lorsqu'une activité stimulante est présente. Selon le type de cellules présentes dans les colonies, on distingue des progéniteurs granuleux et/ou macrophagiques (colony-forming unit granulocyte ou CFU-G, colony-forming unit macrophage ou CFU-M, colony-forming unit granulocyte-macrophage ou CFUGM), des progéniteurs érythroïdes (burst-forming unit erythrocyte ou BFU-E et colony-forming unit erythrocyte ou CFU-E), des progéniteurs mégacaryocytaires (CFU-Meg ou CFU-MK) et des progéniteurs exprimant plusieurs potentialités (colony-forming unit granulocyte erythrocyte macrophage megakaryocyte ou CFU-GEMM ou CFU-Mix). L'élaboration de ces modèles expérimentaux a eu des conséquences scientifiques considérables : l'observation qu'une activité stimulante était indispensable à l'apparition de colonies a abouti à l'identification de plusieurs facteurs de croissance de l'hématopoï̀se incluant le G-CSF et le GM-CSF qui ont maintenant atteint le stade de l'utilisation thérapeutique. Cependant, il est clair que ces tests ne mettent pas en évidence les cellules souches primitives : les CFU sont des cellules qui expriment des potentialités de différenciation réduites et qui n'ont pas de capacité d'autorenouvellement. Par ailleurs, ces conditions de culture ne permettent pas l'étude de la différenciation lymphoïde pour laquelle les cultures liquides sont mieux adaptées.

D'autres tests ont été élaborés, mettant en évidence des colonies plus primitives : il s'agit des HPP-CFC (high-proliferative potential colony-forming cell) et des blast-CFC. Les HPPCFC sont des colonies de grande taille contenant 500000 cellules ou davantage ; elles sont identifiables à l'œil nu, après trois à quatre semaines de culture, et contiennent essentiellement des macrophages, parfois associés à d'autres cellules myéloïdes [8] ; les HPP-CFC sont résistantes aux substances dont l'action dépend du cycle cellulaire, telles que le 5-fluorouracile. Les blast-CFC [9] sont des colonies de petite taille ( 18 à 100 cellules rondes et réfractiles) apparaissant en trois à quatre semaines, en méthyl cellulose, en présence de faibles concentrations de sérum (2\%). Lorsqu'une blast-CFC est prélevée à l'aide d'une micropipette et cultivée à nouveau dans des conditions similaires, de nouvelles colonies similaires et des colonies secondaires (CFU-GM, CFU-GEMM) apparaissent : cet auto-renouvellement, associé à l'aspect morphologique immature des cellules et à la présence de cellules appartenant à plusieurs lignées dans les colonies secondaires, suggère que la population des blast-CFC et celle des cellules souches hématopoïétiques sont partiellement identiques. Les blast-CFC ont été identifiées dans la moelle osseuse, dans le sang de cordon et dans le sang périphérique. Compte tenu de leur hétérogénéité, ces populations cellulaires sont vraisemblablement en partie superposables et contiennent peut-être une partie des cellules souches.

L'utilisation des cultures médullaires à long terme, initialement décrites chez la souris et adaptées aux cellules humaines, permet de détecter des cellules plus primitives. Lorsque des cellules de moelle osseuse sont cultivées en présence de sérum de veau fœtal, de sérum de cheval et d'hydrocortisone, elles donnent naissance à une population cellulaire adhérente au flacon; cette "sous-couche» reproduit partiellement la diversité cellulaire et fonctionnelle du microenvironnement médullaire et est indispensable au maintien de progéniteurs hématopoïétiques (surtout de type granulo-macrophagiques) pendant quelques semaines. La radiosensibilité différente des cellules stromales (de la sous-couche) et des cellules hématopoïétiques offre l'opportunité de détruire les dernières tout en respectant les premières. Il est alors possible de tester la prolifération et la différenciation de différentes populations cellulaires sur ces sous-couches irradiées : la production de progéniteurs clonogéniques pendant les semaines qui suivent l'initiation des cultures reflète la présence dans l'inoculum de cellules capables de remettre en route l'hématopoïèse dans les cultures médullaires médullaires à long terme, ou LTC-IC (long term culture-initiating 


\section{RÉFÉRENCES}

11. Baum CM, Weissman IL, Tsukamoto AS, Buckle AM, Péault B. Isolation of a candidate human hematopoietic stem cell population. Proc Nall Acad Sci USA $1992 ; 89$ : 28048.

12. Lapidot T, Pflumio F, Doedens M, Murdoch B, Williams DE, Dick JE. Cytokine stimulation of multilineage hematopoiesis from immature human cells engrafted in from immature human cells engrafted
SCID mice. Science $1992 ; 255$ : $1137-41$.

13. Srour EF, Zanjani ED, Cornetta K, et al. Persistence of human multilineage self-renewing lymphohematopoietic stem cells in chimeric sheep. Blood $1993 ; 82$ : 3333-42.

14. Mayani H, Dragowska W, Lansdorp PM Lineage commitment in human hematopoiesis involves asymmetric division of multipotent progenitors and does not appear to be influenced by cytokines. I Cell Physiol 1993 ; $157: 579-86$.

15. Civin CL, Strauss LC, Brovall C, Fackler MJ, Schwartz JF, Shaper JH. Antigenic analysis of hematopoiesis. III. A hematopoietic progenitor cell surface antigen defined by a monoclonal antibody raised against KG-1a cells. J Immunol 1984 ; 133 : $157-65$.

16. Andrews RG, Bryant AM, Bartelmez SH, et al. CD $34^{+}$marrow cells devoid of $\mathrm{T}$ and $\mathrm{B}$ lymphocytes reconstitute stable lymphopoiesis and myelopoiesis in lethally irradiated allogeneic baboons. Blood 1992 ; 80 : 1693-701.

17. Simmons PJ, Torok-Storb B. CD34 expression by stromal precursors in normal human bone marrow. Blood 1991 ; 78 : 2848 53.

18. He XY, Antao VP, Basila D, Marx JC, Davis $B R$. Isolation and molecular characterization of the human CD34 gene. Blood $1992 ; 79$ : 2296-302.

19. Simmons DL, Satterwaithe AB, Tenen DG, Seed B. Molecular cloning of a cDNA encoding CD34, a sialomucin of human hematopoietic stem cells. I Immunol 1992 ; 92 : $267-71$

20. Baumhueter S, Singer MS, Henzel W, et al. Binding of L-selectin to the vascular sia- cells). Le groupe de Eaves a proposé que le nombre de LTC-IC. soit estimé par la production de CFU-GM à la cinquième semaine des cultures, et déduit de ses résultats que chaque LTC-IC est à l'origine de 4CFU-GM [10]. Les limites de ce modèle expérimental résident dans sa brièveté, puisque la reconstitution hématopoïétique est mesurée après un délai bref comparé à la situation physiologique, et dans le caractère déséquilibré de l'hématopoïèse observée, puisque la production de granulocytes et de macrophages prédomine sur la production d'éry- throcytes, de mégacaryocytes et de lymphocytes l'assimilation des LTC-IC et des cellules souches hématopoïétiques est par conséquent discutable. La présence de LTC-IC a été démontrée dans le sang périphérique comme dans la moelle osseuse.

Enfin, plus récemment, sont apparus des modèles expérimentaux cherchant à reproduire la différenciation hématopoïétique humaine in vivo chez des animaux hôtes acceptant la greffe de tissus humains : ce sont les animaux «humanisés". Dans certaines situations de déficit ou d'immaturité du système immunitaire d'un animal, il est possible d'implanter des tissus humains sans que se produise un phénomène de rejet. Ainsi chez les souris SCID - atteintes d'un déficit imṃunitaire $\mathrm{T}$ et $\mathrm{B}$ sévère (severe combined immune deficiency) il est possible d'implanter un fragment de foie foetal, de thymus ou d'os qui perdure ; celui-ci constitue un micro-environnement favorable à l'hématopoïèse humaine, et il est possible de tester la présence de pro géniteurs hématopoïétiques humains dans une population cellulaire par sa capacité de peupler de tels implants [11] : c'est le modèle de la souris SCID humanisée chez laquelle jus qu'à $2 \%$ des cellules circulantes sont d'origine humaine, de nature $B$ essentiellement. Il existe une variante de ce modèle expérimental où l'administration systémique répétée de cytokines humaines permet le maintien à long terme d'une population minoritaire de progéniteurs hématopoïétiques humains circulants, en l'absence de fragment de tissu implanté [12]. Srour et al. [13] ont implanté chez le foetus de mouton, par voie intrapéritonéale, des cellules médullaires humaines ; à la naissance, un certain nombre d'animaux présentaient un faible pourcentage de progéniteurs lymphoïdes et myéloïdes humains dans leur tissu hématopoïétique. L'utilisation de ces modèles a permis de démontrer la présence de cellules reconstituant l'hématopoïèse dans la moelle osseuse et dans le foie foetal et, plus récemment, dans le sang périphérique [7].

Tous ces modèles expérimentaux permettent de tester l'influence des cytokines sur le comportement des progéniteurs hématopoïétiques : les facteurs de croissance (interleukine3, M-CSF, G-CSF, GM-CSF), les facteurs synergiques comme le ligand de c-kit (stem cell factor ou mast cell growth factor), l'interleukine-l ou l'interleukine-6, les inhibiteurs comme les interférons, le transforming growth factor (TGFß), le tumor necrosis factor (TNF $\alpha$ ) ou la macrophage inhibitory protein (MIP-l $\alpha$ ) interagissent au sein d'un réseau complexe de signaux régulateurs, grâce à des récepteurs spécifiques dont l'expression est réglée en fonction du stade de différenciation de la cellule. La réponse aux différentes cytokines des progéniteurs hématopoiétiques du sang périphérique ne paraît pas fondamentalement différente de celle qui a été décrite pour les progéniteurs médullaires. L'étude de l'expression des récepteurs devrait permettre de mieux explorer cette question. En tout état de cause, il ne semble pas exister de cytokine agissant spécifiquement sur les CSSP.

In vitro, les facteurs de croissance de l'hématopoïèse induisent la prolifération des progéniteurs hématopoïétiques, mais leur effet sur les cellules les plus immatures semble limité [14]. I es cellules souches hématopoïétiques de la moelle osseuse sont des cellules au repos, comme le démontre la fréquence élevée des LTCIC dans la population des cellules qui incorporent faiblement la rhodamine 123 [5], et sont résistantes aux substances dont l'action dépend du cycle cellulaire, telles que le 5-fluorouracile et les dérivés du cyclophosphamide. Ces dernières propriétés pourraient être expliquées par l'expression du gène de résistance aux médicaments $(M I) R)$. Le recours à une chimiothérapie myéloablative 


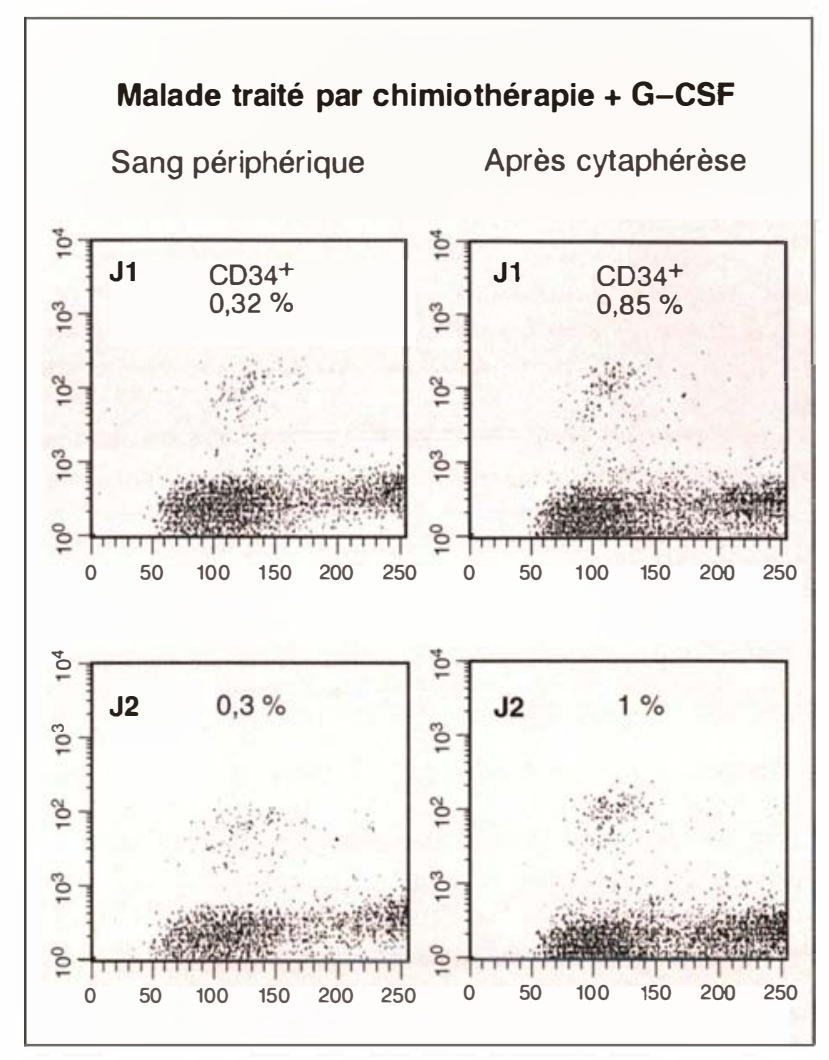

Figure 1. Les cellules CD34+ apparaissent comme une population minoritaire dans le sang périphérique (à gauche) et dans les produits de cytaphérèse correspondants (à droite) collectés pendant deux jours consécutifs, chez un patient à la sortie de la phase d'aplasie chimio-induite, et ayant reçu un facteur de croissance de l'hématopoièse, le G-CSF. Bien que quantitativement faible, cette population est suffisante pour permettre le prélèvement d'un produit cellulaire assurant la reconstitution de l'hématopoièse après chimiothérapie myéloablative. Immunofluorescence directe lanticorps anti-CD34 HPCA-2, clone 8G12, directement couplé à la fluorescéine, analyse par cytométrie en flux).

ou à un facteur de croissance hématopoiétique pour recruter les CSSP induit l'entrée en cycle d'une partie au moins des progéniteurs hématopoïétiques et il est vraisemblable que les CSSP diffèrent des cellules souches hématopoïétiques de la moelle osseuse par leur activité dans le cycle cellulaire, moins en raison de leurs propriétés intrinsèques qu'en raison du mode de prélèvement. Le statut au repos des cellules souches hématopoïétiques semblant être étroitement lié à leur capacité de reconstituer l'hématopoïèse et de s'autorenouveler (si toutef ois on accepte cette dernière hypothèse), il n'est pas certain que les CSSP puissent être substituées aux greffons médullaires dans toutes les situations cliniques, en particulier en situation allogénique ; il sera très important de définir la contribution des CSSP à la reconstitution hématopoïétique à long terme, lorsque les outils nécessaires pour cette évaluation seront opérationnels.
Les progéniteurs hématopoïétiques humains peuvent être identifiés grâce à l'expression d'antigènes de surface parmi lesquels l'antigène CD34

L'immunisation de souris avec une lignée leucémique myéloïde humaine, KGila, a abouti à la génération du premier anticorps monoclonal (Myl0) qui reconnaît une glycoprotéine de 115 kDa d'expression étroite [15]. De nombreux autres anticorps reconnaissant la même glycoprotéine ont été produits par la suite (12.8, 8(12, IMU133, QBEND10, BI3C5...), et ont une spécificité pour d'autres épitopes de la molécule CD34. La population $\mathrm{CD}_{34}{ }^{+}$contient les progéniteurs clonogéniques; elle contient également la population des cellules reconstituant l'hématopoïèse comme cela a pu être démontré en tirant parti de la réactivité croisée de l'anticorps 12.8 avec les cellules du babouin [16]. I es progéniteurs du stroma de la moelle osseuse expriment CD34, suggérant une origine com- mune aux cellules hématopoḯtiques et au micro-environnement médullaire [17]. CD34 est une molécule qui intervient probablement dans l'interaction des progéniteurs hématopoïétiques avec leur environnement comme le suggèrent la structure de la molécule, la localisation du gène sur le bras long du chromosome 1, au sein de plusieurs gènes codant pour des molécules d'adhérence $[18,19]$ et la publication récente d'un travail démontrant que CD34, exprimé sur les cellules endothéliales, liait la L-sélectine ( $\left.m / s n^{\circ} 1, v o l .10, p .124,[20]\right)$. CD34 est exprimé sur les cellules souches hématopoïétiques provenant de la moelle osseuse, du sang périphérique, du sang de cordon et du foie fotal, mais la fréquence de cette population varie selon la source considérée : à l'état basal le pourcentage de cellules exprimant CD34 dans le sang périphérique est de l'ordre de $0,1 \%$ contre $2 \%$ à $5 \%$ dans la moelle (figure 1). Cependant, la population CD $34^{+}$est hétérogène 


\section{RÉFÉRENCES}

21. Terstappen LWMM, Huang S, Safford $M$, Lansdorp PM, Loken MR. Sequential generations of hematopoietic colonies derived from single non lineage committed CD $34^{+}$ CD38- progenitor cells. Blood 1991; 77: 1218-27.

22. Craig W, Kay R, Cutler RL, Lansdorp PM. Expression of Thy-l on human hematopoietic progenitor cells. J Exp Med 1993 ; $177: 1331-42$.

23. Verfaillie C, Blakolmer $K$, McGlave $P$. Purified primitive human hematopoietic progenitor cells with long-term in vitro repopulating capacity adhere selectively to irradiated bone marrow stroma. I Exp Med $1990 ; 172$ : 509-20.

24. Andrews RG, Singer JW, Bernstein ID. Human hematopoietic precursors in longterm culture : single CD34 ${ }^{+}$cells that lack detectable T cell, B cell and myeloid cell antigens produce multiple colony-forming cells when cultured with marrow stromal cells. J Exp Med 1990 ; 172 : 355-58.

25. Bender JG, Unverzagt KL, Walker DE, et al. Identification and comparison of CD34positive cells and their subpopulations from normal peripheral blood and bone marrow using multicolor flow cytometry. Blood 1991 ; $77: 2591-6$.

26. Haas R, Ehrhardt R, Witt B, et al. Autografting with peripheral blood stem cells mobilized by sequential interleukin-3/granulocyte-macrophage colony-stimulating factor following high-dose chemotherapy in non-Hodgkin's lymphoma. Bone Marrow Transplant $1993 ; 12$ : 643-9.

27. Rich KC, Richman CM, Meijas E, Daddona $\mathrm{P}$. Immunoreconstitution by peripheral blood leucocytes in adenosine deaminase-deficient severe combined immunodeficiency. J Clin Invest 1980 ; 66 : 389-95.

28. Bender JG, Williams SF, Myers S, et al. Characterization of chemotherapy mobilized peripheral blood progenitor cells for use in autologous stem cell transplantation. Bone Marrow Transplant 1992 ; 10 : 281-5.

29. Gianni AM, Sienna S, Bregni M, et al. Granulocyte-macrophage colony-stimulating factor to harvest circulating haematopoietic stem cells for autotransplantation. Lancet 1989 ; ii : $580-4$.

30. Bensinger W, Singer J, Appelbaum F, et al. Autologous transplantation with peripheral blood mononuclear cells collected after administration of recombinant granulocyte colony stimulating factor. Blood $1993 ; 81$ :
[5], et les efforts des hématologistes se sont portés sur la recherche de sous-populations susceptibles de définir plus précisément les cellules souches hématopoïétiques, et en particulier de clairement les séparer des cellules clonogéniques. La population des cellules souches hématopoïétiques ne représente qu'une fraction minoritaire - de l'ordre de $1 \%$ des cellules $\mathrm{CD}_{34}{ }^{+}$, sous-population qui peut être caractérisée par l'absence d'expression de l'antigène CD38 [21], par la faible expression de l'antigène Thyl [22], par l'absence ou la faible expression des antigènes de classe II du complexe majeur d'histocompatibilité (HL.A-DR) [23] et par l'absence d'expression d'antigènes caractérisant l'engagement vers la différenciation dans une lignée (caractère lineage negative) $[5,16,24]$. I] ne semble pas que les CSSP diffèrent fondamentalement des cellules souches hématopoïétiques en terme de phénotype [25]; cependant, la faible expression parmi les cellules $\mathrm{CD}_{34}{ }^{+}$d'antigènes spécifiques de la lignée B comme CD19 [26] suggère une maturation différente des cellules du sang par rapport aux cellules médullaires ou une répartition différente des populations de progéniteurs dans le sang et la moelle osseuse.

Le sang périphérique est de plus en plus utilisé comme source de progéniteurs pour les greffes de cellules souches hématopoiétiques en thérapeutique humaine

Chez l'homme, les applications thérapeutiques des cellules souches hématopoiétiques sont déjà anciennes ; initialement l'utilisation de la moelle osseuse a été privilégiée mais, depuis la fin des années 1980, les CSSP font l'objet d'un nombre croissant de programmes d'autogreffe dans le cadre d'intensification de chimiothérapie. Ces développements sont liés à la possibilité de mobiliser de grandes quantités de progéniteurs hématopoïétiques dans le sang périphérique. En effet, si la collecte des CSSP chez un sujet non stimulé n'est pas impossible, elle représente une cytaphérèse d'une durée telle que son application n'est pas envisageable à large échelle [27].

Après une chimiothérapie aplasiante, la réapparition de polynucléaires neutrophiles dans le sang périphérique coïncide avec l'augmentation de la fréquence des progéniteurs hématopoïétiques [28]. La collection devient alors possible lors d'une séance de cytaphérèse d'une durée semblable à celles réalisées pour collecter des plaquettes ou des granulocytes. L'administration de G-CSF ou de GM-CSF à un sujet ne présentant pas de déficit de son hématopoïèse entraîne une augmentation des neutrophiles circulants mais également des progéniteurs hématopoiétiques $[29,30]$. Cette procédure a été largement utilisée en situation autologue, et l'efficacité des deux molécules GCSF et GM-CSF en terme de reconstitution hématopoïétique semble équivalente [31]. L'innocuité apparente de l'administration au long cours de facteurs de croissance de l'hématopoïèse à des patients présentant une neutropénie chronique et l'absence de néoplasie induite dans des systèmes d'hyperexpression artificiels tels que les souris transgéniques ont amené à proposer des protocoles prévoyant de prélever les CSSP chez des donneurs sains après stimulation par le G-CSF, dans l'optique d'une greffe syngénique ou allogénique [32]. Enfin, outre le G-CSF et le GMCSF, d'autres molécules pourraient démontrer leur utilité dans cette situation, en particulier l'interleukine3 [26] et le ligand de c-kit.

La combinaison des deux techniques (administration d'un facteur de croissance de l'hématopoïèse après une chimiothérapie myéloablative) permet d'augmenter le nombre des progéniteurs circulants de façon plus importante. Elle représente le mode de stimulation le plus utilisé pour les patients présentant une maladie maligne dont la progression n'est pas influencée par les facteurs de croissance de l'hématopoïèse $[26,33]$.

Les cellules souches du sang périphérique contribuent à la reconstitution de l'hématopoïèse chez les patients ayant reçu une chimiothérapie aplasiante comme part d'un programme d'intensification thérapeutique, dans le cadre du traitement de cancers ou d'hémopathies

Parmi les hémopathies, les résultats concernant des myélomes, des leucémies aiguës lymphoblastiques et non 
lymphoblastiques, des lymphomes malins hodgkiniens et non hodgkiniens ont été rapportés dans la littérature. La leucémie myéloïde chronique représente un cas particulier : dans la phase chronique de cette maladie, le nombre de progéniteurs hématopoïétiques circulants est considérablement élevé, ce qui autorise un prélèvement en l'absence de stimulation. Cette maladie est constamment fatale, de sorte qu'en l'absence de possibilité de greffe allogénique, une greffe autologue avec des CSSP prélevées en phase chronique et cryopréservées puisse être proposée. Enfin, de nombreuses tumeurs solides, parmi lesquelles les cancers du sein inflammatoires ou métastatiques, les cancers de l'ovaire, les neuroblastomes, les tumeurs germinales, font l'objet de protocoles incluant une greffe de CSSP.

L'appréciation de la contribution des CSSP à la reconstitution de l'hématopoï̀se après chimio- et/ou radiothérapie se heurte à plusieurs obstacles. Dans un premier temps, les CSSP ont été utilisées comme "adjuvant» à la greffe de moelle, soit pour démontrer la faisabilité du prélèvement et de sa réinjection, soit pour limiter la durée de la cytopénie associée à la transplantation médullaire autologue [31, 34]. Secondairement, les CSSP ont été utilisées comme seule source de progéniteurs hématopoïétiques. L'appréciation des résultats (notamment à long terme) est difficile en raison de l'absence d'essai comparatif entre les CSSP et la greffe de moelle autologue, et de l'absence d'outil biologique permettant de mesurer la participation des cellules du greffon à la reconstitution hématopoïétique. En outre, le développement de l'utilisation des CSSP a permis le développement des intensifications séquentielles de la chimiothérapie : ces schémas comportent plusieurs cures de chimiothérapie, aplasiante mais non myélo-ablative, au décours desquelles les malades reçoivent une réinjection de CSSP qui contribuent à réduire la phase de cytopénie, et à permettre de procéder rapidement à la cure suivante [34] ; dans ce contexte, il ne s'agit pas de reconstituer durablement l'hématopoïèse. Au total, seul le développement d'outils biologiques permettant de marquer et donc de suivre le devenir des cellules du greffon $[3,4]$, l'utilisation des CSSP en situation allogénique, et l'expérience croissante des équipes de transplantation permettront de définir les avantages et les inconvénients liés à l'utilisation des CSSP en thérapeutique humaine.

Dans l'état actuel de nos connaissances, la comparaison des résultats observés à ceux obtenus chez les malades bénéficiant d'une greffe de moelle (contrôles historiques, séries appariées) montre cependant que la reconstitution hématopoïétique survient dans un délai identique ou inférieur à celui observé lors des greffes de moelle [31, 36-38]. Cette rapidité dans la reconstitution hématopoïétique concerne aussi bien les polynucléaires neutrophiles que les plaquettes; elle se traduit par une tendance au raccourcissement de la durée de séjour des patients. Il est vraisemblable que ces résultats, s'ils se confirment, se traduiront à terme par une réduction des coûts associés à la procédure de transplantation, même en tenant compte du prix de la collection [1]. Malgré certaines divergences, les résultats rapportés en terme de rechute et de survie semblent actuellement identiques pour les patients bénéficiant d'une greffe de moelle, et pour ceux bénéficiant d'une greffe avec des CSSP.

Quel est le nombre de cellules souches du sang périphérique qui assure une reconstitution de l'hématopoïèse?

Cette question n'est pas propre aux CSSP, et n'a pas reçu de réponse satisfaisante malgré le nombre croissant de malades transplantés depuis vingt ans. La difficulté d'y répondre tient à la fois aux outils utilisés pour dénombrer les cellules souches hématopoïétiques présentes dans le greffon et à ceux utilisés pour mesurer la reconstitution de l'hématopoï̀se. En l'absence de définition précise de la cellule souche hématopoïétique humaine, la "quantification" des greffons repose sur deux paramètres : la numération des cellules clonogéniques (essentiellement les CFU-GM) et celle des cellules $\mathrm{CD}^{2} 4^{+}$. Les CFU-GM sont issues de la division des cellules souches hématopoïétiques, les cellules $\mathrm{CD} 34^{+}$représentent une population hétérogène au sein de laquelle les cellules souches hématopoïétiques représentent une fraction inférieure à $1 \%$. Ces limites expliquent, d'une part, les difficultés à mettre en évidence une relation claire et univoque entre ces deux paramètres (en d'autres termes à savoir si ces deux tests sont équivalents), d'autre part, à connaître le nombre optimal de CFU-GM ou de cellules CD $34^{+}$à réinjecter à un patient pour assurer une sortie d'aplasie dans les meilleurs délais. Les limites théoriques inhérentes à ces tests suggèrent que leur optimalisation et leur standardisation ne permettront pas de lever toutes les ambiguïtés. La réponse à cette situation réside peut-être dans le transfert vers la pratique clinique de tests plus complexes mais mesurant de façon plus précise le contenu en $\mathrm{CSH}$ des greffons. Il pourrait s'agir de tests fonctionnels, tels la mesure des I.TCIC, ou de la numération d'une population dont le phénotype définit plus étroitement la population des progéniteurs hématopoïétiques immatures (par exemple la population $\mathrm{CD}_{34}{ }^{+}$/ CD38-).

La seconde difficulté tient à la notion même de reconstitution hématopoiétique. Dans une optique médicale, l'élément important est la réapparition des polynucléaires neutrophiles et, à un degré moindre, des plaquettes, deux éléments qui conditionnent la sortie du patient d'un secteur d'hospitalisation conventionnel. Ces événements surviennent habituellement dans un délai mesuré en jours. Du point de vue du scientifique, il s'agit là d'une reconstitution à court terme qui ne préjuge pas de la reconstitution à long terme de l'hématopoïèse, laquelle se mesure en mois et en années. Les études réalisées chez la souris montrent que ce ne sont pas les mêmes populations de progéniteurs qui sont impliquées dans ces phénomènes. En réalité, il est vraisemblable que les véritables cellules souches hématopoḯtiques n'interviennent pas dans la sortie immédiate d'aplasie, phénomène dont seraient responsables des progéniteurs beaucoup plus mûrs, proches des CFU-GM [34-36]. Finalement, seule notre capacité à clairement identifier les cellules originaires du greffon et les cellules originaires du receveur permettra d'établir la contribution des CSSP à la reconstitution, à long terme et à 
court terme, de l'hématopoïèse, et de savoir si ces événements sont influencés par la quantité de cellules réinjectées.

Le prélèvement de cellules souches hématopoïétiques peut contenir des cellules tumorales résiduelles

Ces cellules indésirables sont le plus souvent présentes à un niveau indétectable par les techniques habituelles lorsque le sang d'un patient est prélevé en vue d'une transplantation autologue. Le rôle de ces cellules tumorales dans la rechute suivant une autogreffe reste contesté, malgré les expériences récemment rapportées de "marquage" des cellules du greffon par transfert de gène [38] ; il ne peut être exclusif puisque des rechutes sont observées en situation allogénique. Cependant, l'hypothèse selon laquelle les CSSP pourraient être indemnes de contamination tumorale dans certaines situations pathologiques (ou pour le moins pourraient être "contaminées" par un nombre moins important de cellules tumorales) a été avancée pour justifier l'emploi de ces cellules de préférence à un greffon médullaire [39]. Cette hypothèse est remise en question par plusieurs publications récentes mettant en évidence la présence, et même la mobilisation, des cellules tumorales lors de la préparation au prélèvement par cytaphérèse [41]. Cependant, le caractère "fonctionnel » de ces cellules tumorales n'a pas été étudié : s'agit-il de cellules évoluant vers la mort cellulaire ou de cellules susceptibles de se diviser et d'être à l'origine d'une rechute de la maladie sous-jacente? Il n'existe pas actuellement de démonstration formelle d'un bénéfice à l'usage des CSSP de ce point de vue. L.es conséquences de la présence de cellules tumorales résiduelles pourraient être modifiées par l'utilisation de procédés permettant de sélectionner les progéniteurs hématopoïétiques, à l'exclusion des autres populations cellulaires (voir plus loin). Il s'agit en tout état de cause d'un problème crucial lorsque l'on envisage de manipuler in vivo les cellules hématopoïétiques.

La présence de cellules $T$ en grand nombre sera-t-elle à l'origine d'une fréquence accrue de maladie du greffon contre l'hôte lorsque les cellules souches du sang périphérique seront utilisées en situation allogénique ?

I.es produits de cytaphérèse contiennent une quantité de lymphocytes $T$ qui est environ dix fois supérieure à ce qu'elle est dans un greffon médullaire. Lors d'une greffe médullaire allogénique, ces cellules sont à l'origine de la maladie du greffon contre l'hôte (graft-versus-host disease, GVHD) ; il s'agit d'une complication immunologique redoutable au cours de laquelle les cellules immunocompétentes injectées avec le greffon reconnaissent les tissus du receveur qui n'est plus protégé par son propre système immunitaire, détruit lors du conditionnement à la greffe. Bien que l'incidence et la sévérité de cette complication aient été réduites par l'utilisation d'immunosuppresseurs puissants tels que la ciclosporine, la GVHD reste à l'origine d'une part importante de la morbidité et de la mortalité associées à la greffe allogénique. Il est donc essentiel de s'assurer que les CSSP ne seront pas à l'origine d'une augmentation de la fréquence et de la gravité de la GVHD. I.es résultats préliminaires rapportés lors de récents congrès semblent cependant rassurants, les quelques patients traités n'ayant pas présenté de GVHD grave.

Deux commentaires s'appliquent aux cellules immunocompétentes comme ils s'appliquaient aux cellules tumorales résiduelles : d'une part, les procédés de sélection des progéniteurs hématopoïétiques devraient permettre de réduire le nombre de lymphocytes associés, d'autre part, il est nécessaire de clairement identifier les deux populations si l'on envisage des manipulations $e x$ vivo.

Il est possible de sélectionner les seules cellules exprimant l'antigène CD34 parmi les cellules collectées par cytaphérèse

La sélection des cellules exprimant CD34 peut être réalisée par de multiples méthodes dont certaines ont actuellement atteint le stade de l'utilisation clinique. Il s'agit de systèmes d'immunosélection utilisant un anticorps anti-CD34 fixé à un support inerte ou à des billes magnétiques, et 

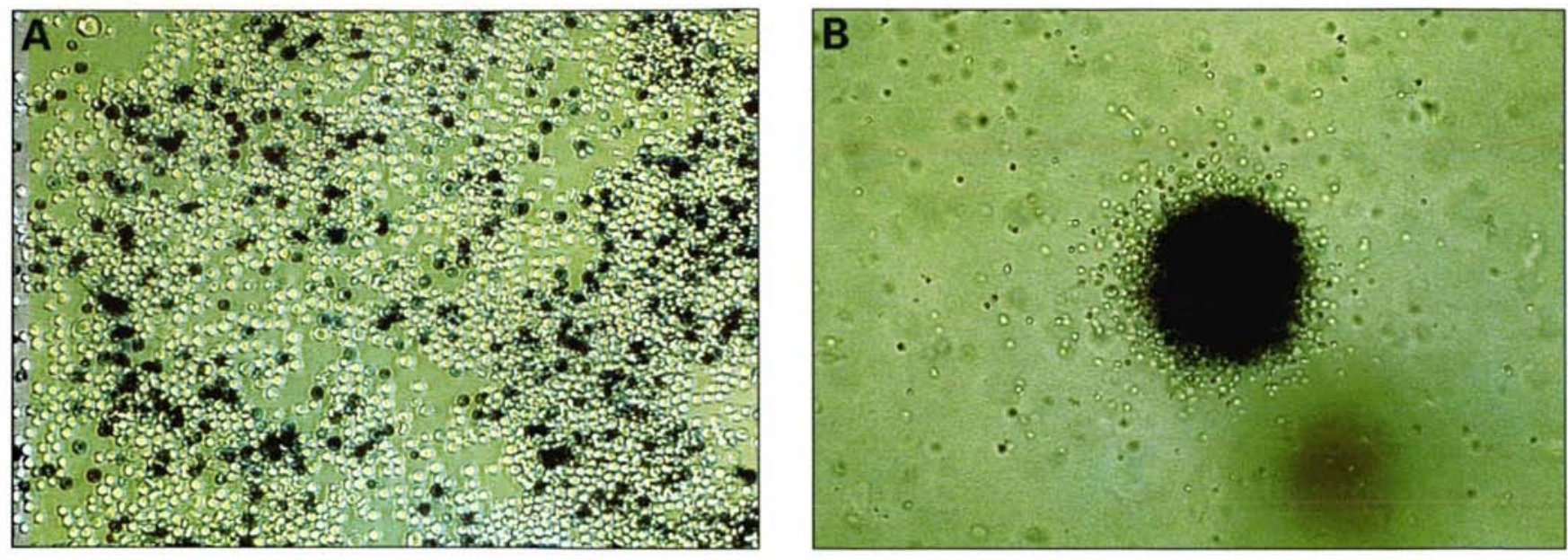

Figure 2. A. Infection de cellules $C D 34^{+}$du sang périphérique humain par coculture avec la lignée productrice de rétrovirus amphotrope, A7.21. Le rétrovirus, MFG-nIsLacZ, contient un gène codant pour une version modifiée de la $\beta$ galactosidase d'Escherichia coli, nls LacZ, dont la localisation est nucléaire, et dont l'activité peut être détectée par la coloration au X-gal. B. Une colonie contient des cellules transduites par le même rétrovirus. (crédit photographique : Anne-Marie Imbert et Claude Bagnis.)

qui permet de séparer les cellules CD $34^{+}$des cellules CD34- [42]. Ces systèmes permettent de sélectionner les cellules CD34+ dans la moelle osseuse, les produits de cytaphérèse ou le sang de cordon.

Les avantages théoriques liés à la "purification" de cellules $\mathrm{CD} 34^{+}$ sont multiples : facilité de définition, de conservation et de manipulation des cellules congelées, absence de sélection des cellules tumorales résiduelles ou des cellules lymphoïdes (déplétion T). Cependant, aucun de ces avantages n'a reçu de confirmation clinique dans l'état actuel des connaissances médicales. L'expérience limitée acquise avec ces technologies montre une reconstitution hématopoïétique survenant dans les délais habituels, mais aucun bénéfice sur la survie ou sur la rechute n'a été démontré [42]. En tout état de cause, l'utilisation de ces systèmes de séparation des cellules exprimant CD34 a un prix : si l'échantillon final est plus concentré en cellules CD $34^{+}$, le nombre absolu de cellules CD $34^{+}$ qui est collecté ne représente que $30 \%$ à $50 \%$ des cellules $\mathrm{CD} 4^{+}$présentes dans le tissu d'origine. Cependant, la maîtrise de cette étape est importante avant d'envisager la manipulation ex vivo des progéniteurs hématopoïétiques.
L'expansion in vitro des progéniteurs hématopoiétiques du sang périphérique peut être réalisée en utilisant des combinaisons de cytokines

La multiplicité des cytokines et de leurs combinaisons offre aux médecins et aux scientifiques l'opportunité d'induire la multiplication des progéniteurs hématopoïétiques in vitro. Deux objectifs doivent cependant être clairement séparés. La production in vitro de cellules différenciées et fonctionnelles - érythrocytes, plaquettes, neutrophiles, monocytes, cellules dendritiques, lymphocytes B et $\mathrm{T}$ - destinées à un usage thérapeutique apparaît comme un objectif réalisable dans un avenir relativement proche. Les avantages de ces thérapeutiques substitutives sont évidents : production adaptée aux besoins des patients, standardisation des produits, diminution des risques de transmission d'agents infectieux (en particulier les agents transmissibles non conventionnels). Aujourd'hui, les conditions nécessaires à la production de chacune de ces populations cellulaires ne sont pas parfaitement définies, mais la production de progéniteurs et de cellules myéloïdes mûres apparaît réalisable [43-45], avec des applications proches dans le domaine de la transplantation. L'utilisation de ces pro- duits cellulaires manipulés, soit en association avec un greffon non manipulé, soit après une chimiothérapie aplasiante mais non myélo-ablative, pourrait se traduire par une réduction de la durée de la cytopénie et des événements indésirables associés. Il n'est alors pas clair si le bénéfice éventuellement obtenu serait lié à l'augmentation du nombre de progéniteurs (une relation qui n'a pas pu être formellement établie pour les greffons non manipulés), à la présence de cellules différenciées et activées par les facteurs de croissance ou aux deux facteurs.

La multiplication in vitro des cellules souches hématopoïétiques représente un autre objectif extrêmement intéressant pour le médecin comme pour le chercheur : elle permettrait de répondre à des objectifs comme le transfert de gène ; la mise en cycle et l'augmentation du nombre des progéniteurs pourraient pallier en partie la difficulté de transduire des cellules souches par des vecteurs rétroviraux (figure 2). Elle offrirait également des espoirs thérapeutiques à des patients chez lesquels il n'est pas possible de collecter un greffon suffisant du fait de la maladie sous-jacente (aplasie médullaire par exemple) ou du fait de traitements antérieurs (patients ayant reçu plusieurs cycles de chimiothérapie anti-néoplasique). Elle 


\section{RÉFÉRENCES}

40. Ross AA, Cooper BW, Lazarus HM, et al Detection and vability of tumor cells in peripheral blood stem cell collections from breast cancer patients using immunocytochemical and clonogenic assay techniques. Blood 1993 ; 82 : 2605-10.

41. Brugger W, Bross KJ, Glatt M, Weber F Mertelsmann R, Kanz L. Mobilization of tumor cells and hematopoietic progenitor cells into peripheral blood of patients with solid tumors. Blood $1994 ; 83$ : 636-40.

42. Berenson RJ, Bensinger WJ, Andrews RG, Buckner CD, Bernstein ID. Transplantation with enriched stem cell fractions. In Wunder EW, Henon PR, eds. Peripheral blood stem cell autografts. Berlin-Heidelberg : Springer-Verlag, 1993: 243-9.

43. Brugger W, Mocklin W, Heimfeld S, Berenson RJ, Mertelsmann R, Kanz L. Ex vivo expansion of enriched peripheral blood expansion of enriched peripheral blood
$\mathrm{CD} 34^{+}$positive progenitor cells by stem cell factor, interleukin-1 beta (IL-1 beta), II -6. IL-3, interferon-gamma and erythropoietin. Blood 1993 ; 81 : 2579-84.

44. Haylock DN, To LB, Dowse TL, Juttner CA, Simmons PJ. Ex vivo expansion and maturation of peripheral blood CD $34^{+}$cell into the myeloid lineage. Blood $1992 ; 80$ 1405-12.

45. McAllister IB, Teepe M, Gillis S, Williams DE. Ex vivo expansion of peripheral blood progenitor cells with recombinant cytokines. Exp Hematol $1992 ; 20$ : 626-8.

46. Verfaillie CM, Miller WJ, Boylan K, McGlave PB. Selection of benign primitive hematopoietic progenitors in chronic myelogenous leukemia on the basis of HIA-DR antigen expression. Blood $1992 ; 79: 1003$ 10.

47. Cassel A, Cottler-Fox M, Doren S, Dunbar CR. Retroviral-mediated gene transfer into CD34enriched human peripheral blood stem cells. Exp Hematol 1993 ; 21 : 585 91.

48. Bregni M, Magni M, Siena S, Di Nicola M, Bonnadonna G, Gianni AM. Human peripheral blood hematopoietic progenitors are optimal targets of retroviral mediated gene transfer. Blood $1992 ; 80: 1418-22$

49. Bagnis C, Gravis G, Imbert AM, et al. Retroviral transfer of the nls-LacZ gene into human CD34+ cell populations and into TF-1 cells : future prospects in gene thera- pourrait être appliquée à des malades leucémiques chez lesquels il serait possible de séparer une population résiduelle normale des cellules leucémiques, comme le suggèrent certaines études sur la leucémie myéloïde chronique [46]. L'expansion in vitro des cellules souches hématopoïétiques suppose que soit clairement identifié un signal (une cytokine ou une combinaison de cytokines ?) qui provoque leur prolifération sans induire leur différenciation. Dans l'état actuel de nos comnaissances, ce signal n'a pas été identifié. I a plupart des résultats rapportés concernant la manipulation $e x$ vivo des cellules CD $34^{+}$du sang périphérique [43-45] suggèrent, bien au contraire, que la prolifération cellulaire s'accompagne d'une modification des propriétés fonctionnelles et phénotypiques, en particulier de la diminution puis de la disparition des cellules CD34+ après quelques jours de culture ; au mieux, il y a maintien de la population des cellules les plus primitives qui apparaissent peu sensibles à l'action des cytokines [14]. La possibilité que soit altérée la capacité de ces cellules manipulées à restaurer l'hématopoïèse, et leur utilisation exclusive comme greffon après une chimiothérapie myélo-ablative, doivent donc être discutées. En l'état actuel de nos connaissances de la physiologie des cellules souches hématopoiétiques, deux éléments suggèrent que l'objectif d'induire leur multiplication in vitro se heurte à des problèmes majeurs : d'une part, il s'agit essentiellement de cellules quiescentes, et nous ne savons pas s'il est possible que ces cellules retournent à l'état de quiescence après une étape de prolifération ; d'autre part, il est vraisemblable que le rôle des cytokines actuellement reconnues n'est pas de contrôler la survie et la prolifération des cellules souches hématopoïétiques primitives.

Les cellules souches du sang périphérique, cibles de manipulations génétiques

I.es cellules souches hématopoïétiques présentent un intérêt majeur comme cible potentielle pour la thérapie génique dans des situations pathologiques aussi diverses que des maladies héréditaires monogéniques comme les hémoglobinopathies et certains déficits immunitaires, ou des maladies acquises comme les hémopathies, les cancers ou le SIDA (figu$r e$ 2). I a difficulté à transduire ces cellules est liée à plusieurs problèmes dont le faible nombre des cellules souches hématopoiétiques et la faible efficacité de transduction des vecteurs actuellement disponibles. I.es vecteurs rétroviraux qui sont aujourd'hui les plus utilisés ne sont intégrés que par des cellules en cycle. D'une part, les cytokines induisent la prolifération des progéniteurs tardifs plus que celle des cellules souches, d'autre part, les cytokines induisent prolifération et différenciation. Il est trop tôt pour savoir si ce paradoxe est compatible avec les objectifs de la thérapie génique. En tout état de cause, des vecteurs susceptibles d'être intégrés par des cellules non en cycle sont théoriquement intéressants. Les adénovirus sont capables d'infecter des cellules quiescentes, mais les cellules hématopoïétiques ne possèdent pas le récepteur approprié ; par ailleurs, le transgène reste sous une forme épi- somique et les cellules transduites sont progressivement "diluées" au cours des divisions cellulaires, ce qui en fait un vecteur moins attirant pour les progéniteurs hématopoiétiques appelés à se diviser fréquemment. L'AAV (adeno-associated virus) a la possibilité d'inf ecter des cellules quiescentes et de s'intégrer dans le génome; il représente donc un vecteur intéressant, mais ses qualités sont à confirmer par l'expérience. I.es vecteurs non viraux, comme les liposomes et les polycations, doivent être améliorés pour espérer l'intégration du transgène à une fréquence compatible avec les applications cliniques. Finalement, quel que soit le vecteur utilisé, l'expression à moyen et long terme du transgène reste un phénomène mal compris et maîtrisé. Plusieurs équipes de recherche travaillent actuellement au développement de vecteurs et de constructions qui permettraient une expression sélective ou inductible du transgène. Cassel et al. ont récemment rapporté la possibilité d'infecter des cellules CD $34^{+}$provenant du sang périphérique de patients atteints de myélome par un rétrovirus contenant le gène de résistance à la néomycine 
[47]. L'efficacité d'infection, en présence d'une combinaison de cytokines, était identique à celle observée avec des cellules provenant de la moelle osseuse [3] ou du sang de cordon. Ils confirmaient ainsi les résultats de Bregni et al. [48] qui suggéraient que seules les cellules obtenues après stimulation du domneur pouvaient être transduites efficacement. Nous avons récemment rapporté la possibilité d'inf ecter ces cellules avec un rétrovirus contenant le gène codant pour la $\beta$-galactosidase (nls-Lac'Z) [49]. Le gène de résistance à la néomycine et le gène codant pour la $\beta$-galactosidase bactérienne sont des gènes dits "rapporteurs", sans fonction thérapeutique, qui permettent d'étudier le devenir des cellules transduites pendant plusieurs mois.

En conclusion, le sang périphérique représente une source intéressante de progéniteurs hématopoïétiques. Les propriétés fonctionnelles et phénotypiques de ces cellules ne semblent pas très différentes de celles des progéniteurs hématopoïétiques de la moelle osseuse. La facilité avec laquelle ces cellules peuvent être recueillies en situation clinique explique le développement très rapide de leur utilisation, et la multiplicité des projets envisagés en terme de manipulation ex vivo incluant le transfert de gène. Cette utilisation croissante et les travaux scientifiques associés devraient permettre de rapidement répondre aux questions qui persistent, en particulier sur la capacité des CSSP de donner naissance aux cellules lymphoïdes B et T, et sur leur contribution au rétablissement de l'hématopoïèse à long terme. A ces conditions, ces cellules deviendront rapidement une composante thérapeutique majeure des thérapies cellulaires somatiques et des thérapies géniques

\section{Summary}

Human peripheral blood stem cells

«Peripheral blood stem cells» (PBSC) usually refer to apheresis products collected in patients primed with chemotherapy and/or recombinant hematopoietic growth factors such as granulocyte-colony stimulating factor (G-CSF) or granulocyte-macrophage-colony stimulating factor (GM-CSF). The proportion of true "stem cells" in these cell populations is very small, much smaller than the frequency of other, more mature progenitors, or of differentiated cells. However, the use of apheresis products has greatly changed the clinical practice of high-dose chemotherapy over the last few years. Harvesting is easy and does not require general anesthesia, as does bone marrow collection. Time to hematopoietic recovery is usually shorter in patients receiving PBSC as compared to patients receiving bone marrow. In association with the use of hematopoietic growth factors during chemotherapy-induced cytopenia, PBSC help reduce morbidity associated with high-dose chemotherapy, and thus allow more patients with poor risk cancer to benefit from this technique. Many scientific questions remain unsolved. Identification of stem cells is still hampered by the lack of a simple and reproducible in vitro assay. These difficulties do not allow to accurately appreciate the contri- bution of different populations of progenitors to hematopoietic recovery, and thus to predict the quality of a hematopoietic graft. The ability of PBSC to repopulate lymphoid lineages is poorly defined. Despite these uncertainties, PBSC have numerous potentialities. Associated with the clinical use of techniques such as selection of normal progenitors (based on the expression of the CD34 antigen for example), or liquid cultures supplemented with recombinant cytokines (the so-called " ex-vivo expansion"), they offer the opportunity to change the balance between normal hematopoietic progenitors on one hand, and residual tumor cells or accessory cells such as $\mathrm{T}$ lymphocytes on the other hand. As a consequence, purging or prevention of graft-versushost disease with $\mathrm{T}$ cell depletion can be envisioned from a new standpoint. Finally, PBSC represent a very interesting target for genetic manipulations. These multiple potentialities explain why the use of apheresis products contributes to broaden the application of hematopoietic stem cell transplantation, a practice that in many cases comes closer to transfusion. It also explains why PBSC already appear as a matter of economic and ethical controversies, being one of the first widely used " cellular therapies".

\section{TIRÉS À PART}

